\title{
El impacto de la adicción al celular o nomofobia en estudiantes universitarios: caso de dos universidades mexicanas
}

\author{
Gladys del Carmen Medina Morales \\ gladys.medina@ujat.mx \\ Universidad Juárez Autónoma de Tabasco \\ Villahermosa, México \\ María Guadalupe Veytia Bucheli \\ draveytiabucheli@gmail.com \\ Universidad Autónoma del Estado de Hidalgo \\ Pachuca, México
}

\section{RESUMEN}

El uso de los dispositivos móviles, como los celulares, ha propiciado una serie de cambios de patrones, conductas, así como fenómenos que impactan en la interacción cotidiana. El objetivo de la presente investigación fue comparar el nivel de adicción al teléfono celular entre los estudiantes de dos universidades mexicanas, la Universidad Autónoma del Estado de Hidalgo (UAEH) y la Universidad Juárez Autónoma de Tabasco. El estudio se realizó durante 2019 e inicios del 2020, a una población total de 1009 estudiantes, 785 eran mujeres y 224 hombres, con una edad promedio de 20 años. Tras un análisis factorial, se determinó tomar aquellas preguntas que midieran dos dimensiones: Emociones y Físico-Social y reportar los resultados del comparativo. Dentro de los principales hallazgos identificados, en general, la nomofobia afecta más a los estudiantes en la dimensión Físico-Social. Se encontró que a los estudiantes de la UJAT les afectaba más en malestares físicos, al no disponer del celular; y personales, al aislarse de las personas cercanas y descuidar algunos asuntos. En la dimensión de emociones no se encontró diferencias significativas entre los dos grupos. Se concluyó que, los estudiantes presentan diferentes niveles de nomofobia, además de que no todos se asumen con este trastorno, ya que son conscientes de que el uso excesivo del móvil los aleja de la realidad, del contexto, y a su vez afecta su rendimiento escolar.

Palabras clave: teléfono móvil; nomofobia; actitud estudiantil; universidad. 


\title{
The impact of cell phone addiction or nomophobia on college students: case of two Mexican universities
}

\begin{abstract}
The use of mobile devices such as cell phones have led to a series of changes in patterns, behaviors, as well as phenomena that impact our daily interaction. The research that was carried out allowed comparing cell phone addiction scores in students of two mexican universities, the first located in the State of Hidalgo, and the second in the State of Tabasco. To carry out the study, during 2019 and 2020, to a total population of 1009 students of which 785 were women and 224 men with an average age of 20 years. After a factor analysis, it was determined to take those questions that measured two dimensions: Emotions and Physical-Social and report the results of the comparison. Among the main findings identified in general, nomophobia affects students more in the Physical-Social dimension. It was found that the UJAT students were more affected by physical discomfort due to not having a cell phone, personal, isolating themselves from close people and neglecting some matters. In the Emotions dimension, no significant differences were found between the two groups. It was concluded that students present different levels of nomophobia, because not all are assumed to have this disorder. They are aware that the excessive use of the mobile phone takes them away from reality, from the context, and in turn affects their school performance.
\end{abstract}

Keywords: cell-phone; nomophobia; student attitudes; university.

Artículo recibido: 30 noviembre. 2021 Aceptado para publicación: 29 diciembre 2021

Correspondencia: gladys.medina@ujat.mx Conflictos de Interés: Ninguna que declarar 


\section{INTRODUCCIÓN}

En la sociedad de este siglo, los procesos comunicativos han evolucionado y diversificado debido al uso de las Tecnologías de la Información y la Comunicación (TIC), lo cual permite acortar distancias, facilitando la comunicación mediada. Esta necesidad comunicativa virtual se presenta en los distintos rangos de edad, pero de manera especial en los jóvenes universitarios (Junquera, 2016). Según la Asociación de Internet MX (2017), el 90 \% de los internautas mexicanos son usuarios de los móvilesEn un estudio sobre los hábitos de los usuarios de Internet en México (Asociación de Internet MX, 2018), la conexión a internet la utilizaban en un $51 \%$ las mujeres y $49 \%$ los hombres. Dentro de los promedios de frecuencia de uso está el grupo de 12 a 17 años con un $18 \%$, el grupo de 18 a 24 años con $17 \%$. Este último corresponde al grupo de edad de los universitarios.

La gran diferencia en el uso de los equipos, en los que destaca el empleo de teléfono inteligente, ha implicado un incremento en los últimos años, debido a su portabilidad y a la baja en los costos del equipo y del servicio, entre las aplicaciones más usadas están las redes sociales[A3]. De acuerdo con García y Fabila (2014); Britos y Brítez (2015); Flores et al (2015); Matoza-Baez y Carballo-Ramírez (2016), ha sustituido a otros dispositivos por su incremento en sus funciones. Todo ello ha propiciado que en la actualidad se hayan vuelto imprescindibles en la vida cotidiana de los jóvenes.

En la actualidad, es casi inusual encontrar a personas que no cuenten con este dispositivo celular. Aunque ello ha implicado una gran diversidad de usos que apoyan la vida cotidiana, también la han afectado. En el ámbito escolar, su uso ha propiciado distracciones que impactan en el rendimiento académico y fomentan el hábito de retrasar las actividades académicas, es decir, la procrastinación, un rasgo que pospone las actividades importantes por aquellas más entretenidas. En consecuencia, usan su dispositivo en el tiempo de trabajo escolar para visitar páginas y utilizar redes sociales y con ello postergar la realización de actividades (Osio et al, 2014).

En relación a lo anterior, Dixit et al (2010), sostuvieron que el grupo más vulnerable a este fenómeno del uso ampliado del celular, es el de la edad escolar, ya que, además de aumentar los distractores, se reducen el rendimiento académico, los niveles de motivación en los procesos de aprendizaje, así como la relación [A6] que se produce entre amigos y familiares, debido a que ocasiona un cansancio mental crónico, 
presentando conductas adictivas. En ese sentido, se ha estudiado el uso excesivo del teléfono celular, como un trastorno psicológico, denominado nomofobia, el cual se deriva del inglés "no-mobile-phone-phobia" (Yildirim, 2014; Quesada y Carballo, 2017). King et al. (2010; 2014), lo definieron como un tipo de fobia situacional, la cual es causada por una reacción intensa que puede ser tanto física como emocional, produce un miedo irracional a salir de casa sin el celular para no interrumpir las conversaciones por teléfono, tanto a través de llamadas, como de la comunicación a través de las redes sociales.

La nomofobia a partir de los resultados identificados por Forgays et al (2014) y RamosSoler et al (2017), ya es objeto de estudio de interés de diversas disciplinas, como la medicina, la educación, la sociología, la psicología, la economía, entre otras, debido a las consecuencias observadas entre los sujetos, que modifican su comportamiento en los distintos contextos en los cuales se desenvuelven. Para estudiarlo, se han diseñado algunos instrumentos que permiten valorar este fenómeno de la actualidad. Una de las escalas más conocidas y empleadas es la que propusieron Yildirim y Correia (2015) realizada en inglés y conformada por 20 ítems, con una escala de medición tipo Likert. La creación de esta escala es reciente, por lo que se han encontrado escasas investigaciones acerca de su aplicación y resultados, pero uno de los principales hallazgos es que se ha adaptado la escala en el idioma español.

Para analizar los resultados obtenidos con la aplicación de dicha escala, se analizaron diversos estudios que la emplearon traducida al idioma español y alguna adaptada. Entre ellos, el de Morales et al (2016), quienes llevaron a cabo una investigación correlacional entre el nivel de nomofobia y el rendimiento académico de los estudiantes de la Universidad San Pedro Sede Chimbote, en la que identificaron una relación de influencia significativa entre estas dos variables.

Ramos-Soler et al (2017), incluyeron un ítem adicional, redujeron la escala de 7 puntos a 5, donde 1 significa totalmente en desacuerdo, y 5 totalmente de acuerdo. La aplicaron a 372 estudiantes de Educación Secundaria Obligatoria en provincia de España. Dentro de sus conclusiones agruparon a los estudiantes en tres grupos: (a) el primero se refiere a los conectados, que se caracterizan por estar siempre conectados, aunque se encuentren con familiares y amigos, reducen sus horas de sueño por estar conversando con sus contactos o revisando las actualizaciones, uno de sus principales hobbies es 
publicar selfies de manera individual y grupal. Identificaron al $27 \%$, en donde 11 de los 21 ítems de la escala tuvieron valores medios superiores en relación a la muestra, por lo que no indican un grado elevado de nomofobia, pero si representan dependencia al móvil; (b) el segundo bloque denominado como los creativos, quienes aseguran que en su tiempo libre no se encuentran sin hacer nada, hacen deporte, van a clases particulares, entre las aplicaciones que utilizan con mayor facilidad están el WhatsApp y el Instragram, pero no lo hacen de forma frecuente. Este grupo estuvo conformado por el $60 \%$ y (c) el tercer bloque denominado los nomofóbicos, en donde se encuentran valores superiores en todos los ítems, no saben qué hacer si no tienen el teléfono celular, privilegian la comunicación por medio del celular que, en persona, tanto con familiares como con amigos, se puede decir que tienen accesos de ansiedad si no cuentan con el dispositivo, y con ello, se reduce su rendimiento académico. Aquí identificaron a $13 \%$, quienes presentaron valores superiores respecto al total de la muestra.

Torres-Salazar et al (2018) utilizaron la adaptación de la encuesta Ramos-Soler et al (2017), para realizar un estudio correlacional entre la nomofobia y los indicadores de inteligencia en estudiantes de educación superior. Sus principales resultados reflejaron que el número de selfies se considera como un indicador de la presencia de nomofobia. Otra adaptación de la escala de Yldirim y Correia (2015) la realizaron GonzálezCabrera et al (2017), quienes la aplicaron con 306 estudiantes de secundaria de 3 instituciones españolas. Entre sus principales conclusiones se encuentra que un sujeto nomofóbico no se reduce únicamente a las personas que tienen comunicación continua a través de un móvil, sino que, además pareciera que estos sujetos tienen una personalidad cuando se comunican por medio del teléfono móvil, y diferente cuando se encuentran en espacios multitudinarios, pues su comportamiento es introvertido y retraído.

El estudio de la nomofobia se ha privilegiado en los niveles de educación básica, principalmente en educación secundaria, sin embargo, son pocas las investigaciones que se han trabajado a nivel de educación superior. Los universitarios presentan conductas semejantes en el uso del celular, es por ello, que surgió el interés de realizar una investigación que permitiera hacer una comparación entre el nivel de nomofobia de los sujetos, a partir de su dependencia, con su rendimiento escolar. Como afirmó Pascual (2015), es importante que en la escuela y las instituciones educativas de nivel superior 
se aborden temáticas de relevancia tecnológica que impactan en la vida de los sujetos, en este caso, en su formación para el desempeño de una profesión.

El objetivo del presente trabajo fue comparar la adicción al celular de los estudiantes de Licenciatura en Ciencias de la Educación de dos instituciones de Educación Superior de México, la Universidad Autónoma del Estado de Hidalgo (UAEH), ubicada en el centro del país, y la Universidad Juárez Autónoma de Tabasco (UJAT), ubicada en el sur.

\section{MÉTODO}

\section{Tipo de estudio}

El enfoque que se utilizó para llevar a cabo esta investigación fue mixto. Tuvo un enfoque deductivo cuantitativo porque se aplicó la prueba t de Student para determinar las diferencias entre las medias de dos grupos, con los datos de la escala estimativa de nomofobia de Ramos-Soler et al. (2017). El enfoque cualitativo se obtuvo con los datos generados en el apartado de observaciones, que se anexó al instrumento. Esta sección permitió a los estudiantes generar comentarios de manera libre en torno a la temática, para que, a partir de los resultados, se pudieran identificar fortalezas y áreas de oportunidad. para trabajar con los estudiantes al contar con elementos para identificar algunos comportamientos que atender, con niveles de acción que se puedan implementar para enfrentar este fenómeno social, y apoyar a los estudiantes para que ello no afecte su desempeño escolar, ya que como señalaron Jasso-Medrano et al (2017), el incremento en su uso aumenta "de manera similar los factores de riesgo ante una conducta no saludable del uso de la tecnología" (p. 2832)

\section{Población de estudio}

La población de estudio se seleccionó de forma no probabilística. Se encuestaron 1009 estudiantes de dos instituciones de educación superior. En la Universidad Autónoma del Estado de Hidalgo respondieron el cuestionario 504 alumnos (94 del sexo masculino y 410 del sexo femenino); y 505 de la Universidad Juárez Autónoma de Tabasco (130 del sexo masculino y 375 del sexo femenino). La edad media de los participantes fue de 20 años con una desviación estándar (DE) de 2.66. Como las poblaciones son asintóticas, 224 hombres y 785 mujeres, se determinó tomar $45 \%$ de la muestra de mujeres (353) para tener el número de participantes equivalentes. La selección de las universidades se dio a través de un convenio de cooperación académica existente entre ellas. 
Se excluyeron las preguntas $1,5,7,9,11,12,16,17,18,19,20$ y 21 después de realizar el análisis factorial, ya que estas preguntas no aportaban relevancia de acuerdo al objetivo de estudio. En consecuencia, se adaptó la escala y se establecieron dos dimensiones de la escala: (a) Emociones, que consta de 4 ítems (2, 3, 4 y 8 ) y mide las emociones de los estudiantes cuando no tienen sus celulares a la mano, ejemplo: me molesto cuando quiero buscar algo desde mi móvil y no puedo, con una fiabilidad de Cronbach de 0.73; (b) Físico-Sociales, que consta de 4 ítems (10, 13, 14 y 15) que midieron la intromisión, así como dificultades académicas o familiares por el uso del smarthphone, por ejemplo: Suelo estar mirando el celular, aunque esté con gente, ya sea la familia o amigos, con una fiabilidad de alfa de Cronbach de 0.71 , tal como se muestra en la Tabla 1.

Tabla 1. Adaptación de la escala de Nomofobia.

\section{- Emociones}

Me molesto cuando quiero buscar algo desde mi móvil y no puedo.

A veces pienso que se me va a acabar la batería del móvil y me pongo furioso.

Si me quedo sin wifi, inmediatamente busco señal para conectarme donde sea.

Me agobio mucho si no tengo el móvil y pienso que alguien quiere comunicarse conmigo.

\section{- Físico - Sociales}

Me despisto con las tareas del colegio/instituto por estar mirando y utilizando mi móvil.

Por estar usando el móvil duermo menos.

Si me llaman o tengo un aviso en el móvil, lo atiendo inmediatamente, aunque esté con mi familia y amigos.

Suelo estar mirando el móvil, aunque esté con la gente, ya sea la familia o amigos.

Fuente: Modificado a partir de partir de Ramos-Soler y col. (2017). 


\section{Procedimiento}

Se desarrolló un formulario para que los participantes contestaran en línea. Fue un desarrollo que constó de dos partes. En la primera, los estudiantes identificaban su institución, la licenciatura a la que pertenecían, semestre, edad y sexo; la segunda, la batería de items que propiciaron esta investigación. Se incorporó un apartado de comentarios generales, para que los sujetos tuvieran el espacio de expresar dudas y comentarios.

El instrumento se contestó en línea, mediante la utilización de una aplicación desarrollada por un experto en tecnología de la Universidad Juárez Autónoma de Tabasco, con la finalidad de que fuera un recurso amigable, de fácil acceso, y con ello lograr un mayor alcance de participación. La gestión de la encuesta estuvo a cargo de dos investigadoras, una en cada sede. Para ello, se solicitó el apoyo de los directivos de las instituciones para compartir la liga del formulario en cada grupo de la muestra. El periodo de aplicación de la encuesta comprendió desde agosto de 2019 a marzo de 2020. El tiempo requerido para contestar fue de aproximadamente $5 \mathrm{~min}$. A cada uno de los participantes se les garantizó confidencialidad y libertad con sus datos y resultados.

\section{Análisis de Datos}

Para el procesamiento de los datos cuantitativos se utilizó el paquete estadístico para ciencias sociales (SPSS, por sus siglas en inglés: Statistical Package for the Social Sciences) (SPSS, 2018) versión 21. Se calcularon medias, desviaciones estándar y correlaciones, posteriormente, los datos se sometieron a prueba en el programa AMOS para determinar la bondad de ajuste de los modelos de medida. Mediante un análisis factorial confirmatorio se verificó el ajuste del modelo de medición a los datos $(\mathrm{X} 2=$ 75.37, $\mathrm{gl}=25, \mathrm{P}=000 ; \mathrm{SRMR}=0.035 ; \mathrm{AGFI}=0.97$ CFI $=0.97 ; \mathrm{AGFI}=0.97$; RMSEA $=0.45)$.

\section{RESULTADOS Y DISCUSIÓN}

Las dimensiones analizadas para este estudio quedaron definidas como (a) Emociones y (b) Físico-social. Las Tablas 2 y 3 muestran el comparativo que se realizó para identificar las diferencias entre los estudiantes de ambas instituciones. 
En la Tabla 2, correspondiente a la dimensión de Emociones, no se asumen diferencias significativas entre los estudiantes de ambas instituciones, es decir, ellos no se sienten afectados emocionalmente por mantenerse alejados de su teléfono inteligente.

Tabla 2. Diferencias entre instituciones por la dimensión Emociones.

\begin{tabular}{lcccc}
\hline & M (DE) & gl & $\boldsymbol{t}$ & P \\
\hline UAEH & $2.31(0.84)$ & 261 & -1.42 & 0.15 \\
\hline UJAT & $2.47(0.78)$ & 238 & -1.65 & 0.09 \\
\hline
\end{tabular}

Fuente: Elaboración propia.

La Tabla 3 corresponde a la dimensión Físico-social, muestra que al utilizar de manera frecuente el celular, los estudiantes de la UJAT son afectados en la salud, su postura física, su relación y contacto con otras personas de forma presencial. Aunque la afectación es identificada y reconocida, no parece tener importancia para ellos, puesto que repiten la conducta, incrementando las horas destinadas al uso del dispositivo, de acuerdo a los resultados de los comentarios generales.

Tabla 3. Diferencias entre instituciones por la dimensión Físico-Social.

\begin{tabular}{lcccc}
\hline & M (DE) & gl & $\boldsymbol{t}$ & $\mathbf{P}$ \\
\hline UAEH & $2.46(0.85)$ & 173 & -1.83 & 0.07 \\
\hline UJAT & $2.43(0.74)$ & 337 & -0.67 & 0.50 \\
\hline
\end{tabular}

Fuente: Elaboración propia.

La Tabla 4 presenta las diferencias que existen por género, en relación a las dimensiones analizadas en este estudio. Los resultados muestran que los hombres son quienes se sienten más afectados tanto en Emociones como en lo Físico-social. Estos hallazgos se contraponen a la investigación realizada por Ruiz-Palermo et al(2015), quienes afirmaron que existen mayores índices de nomofobia en las mujeres que en los hombres, las cuales manifiestan su inquietud al no tener acceso al teléfono celular. Con este hallazgo, es relevante mencionar, que la dinámica del empleo de este dispositivo es cambiante y no es atribuible su mayor uso a un género en particular.

Dimensión Emociones: No existe una diferencia significativa entre los estudiantes de ambas instituciones. Se analizaron aspectos relacionados con la incapacidad de los estudiantes de mantenerse libre de su celular, se molestan, se agobian con la idea de 
ubicarse en un sitio sin señal de wifi, ya que consideraron que algún familiar podría necesitar comunicarse con ellos o bien por imprevistos laborales e inconformidades con la pareja: "Me da miedo no tener el celular debido a que mis padres no se pueden comunicar conmigo para saber si estoy bien, ya que vivo lejos, o para los avisos que mandan al grupo de la escuela y no poder estar informada" (S1).

Los hallazgos de esta investigación, donde los jóvenes argumentaron razones de felicidad al tener su teléfono celular en la mano, se sienten acompañados, seguros y completos si cuentan con su dispositivo cerca, coinciden con Ramos-Soler y col. (2017), quienes, en una investigación realizada a estudiantes de secundaria en España, afirmaron creer que la felicidad depende de tener el teléfono inteligente más moderno y estar comunicados siempre. Asimismo, Chou y Edge (2012) encontraron que a los usuarios de dispositivos móviles y aplicaciones les produce felicidad recibir like de sus contactos conocidos o no. También los resultados son semejantes a los de Ramírez y Vargas (2018), quienes utilizaron la adaptación de la escala realizada por GonzálezCabrera et al (2017), donde observaron puntuaciones estadísticamente significativas entre la relación de nomofobia con la dimensión de renunciar a su comodidad, es decir, ellos prefieren mantener su nivel emocional a partir de su uso. Asimismo, Chou y Edge (2012) encontraron que a los usuarios de dispositivos móviles y aplicaciones les produce felicidad recibir like de sus contactos conocidos o no. Los jóvenes argumentaron razones de felicidad al tener su teléfono inteligente en la mano, se sienten acompañados, seguros y completos si cuentan con su dispositivo cerca.

Se ubicaron a estudiantes, en un menor número, que pueden pasar tiempo sin un teléfono celular, como lo señaló uno: "No siento muy necesario el teléfono cómo para distraerme mucho en él, o verlo como una obsesión" (S2). Estos estudiantes reflejan a partir de sus comentarios que realizan deporte, actividades al aire libre, y que para ellos no es tan importante estar conectados todo el día. Estos resultados coinciden con los de Ramírez y Vargas (2018), en torno a la nomofobia y los rasgos de personalidad de estudiantes de la universidad peruana, Lima Metropolitana, en donde utilizaron la adaptación de la escala realizada por González-Cabrera et al (2017), con la que no se observaron puntuaciones estadísticamente significativas entre la relación de nomofobia con el rasgo de personalidad, pero sí con la dimensión de renunciar a su comodidad, es decir, ellos prefieren mantener su nivel emocional a partir de su uso. 
Dimensión Físico-Social: Los resultados presentan datos de significancia. A los estudiantes de la UJAT es a quienes les afecta más. Se agruparon las razones en tres aspectos:

a) Físicos, como malestar en general, insomnio, sueño arrítmico, dolor de cabeza, pérdida de concentración. Como "Me siento feliz, tranquila. Si no lo tengo, se me sube el ritmo cardiaco" (S3). Al respecto Sánchez-Carbonell et al (2008), afirmaron que una persona que pasa mucho tiempo empleando el teléfono celular, es posible que presente trastornos fisiológicos como cervialgias, tendinitis, síndrome del túnel carpiano y alteraciones de la visión, del sueño y de la alimentación.

b) Personales, los estudiantes expresaron que procuran estar al pendiente de su dispositivo móvil por si surge alguna situación de emergencia familiar o laboral. “Sólo por cuestiones de trabajo trató de observar más mi celular" (S4). "Siento que últimamente paso demasiado tiempo en mi celular y descuido ciertas cosas" (S5). Esto es, están más pendientes de lo que sucede a las personas distantes físicamente que a su entorno cercano. Echeburúa y Corral (2010) destacaron que, el abuso del celular produce aislamiento social y se empieza a generar más comunicación con personas de manera virtual que de forma presencial, por lo que alguien que tenga una personalidad vulnerable y relaciones sociales débiles, son más propensos a hacerse adictos a esta herramienta tecnológica, ya que en el entorno virtual encontrarán recompensas inmediatas, sin embargo, con el pasar del tiempo, se generará un vacío existencial, falta de objetivos, y en ocasiones, el desarrollo de depresión.

c) Académicos, argumentan el uso del celular porque permite el desarrollo, la consulta de traductores, diccionarios, ubicaciones, entre otros. "El celular tiene límite y su función es apoyar en lo que no puede resolver investigaciones, modificación de textos, etcétera." (S6). De acuerdo a la investigación presentada por Ramos-Galarza et al (2017), encontraron por una parte que el uso del internet es un factor que en la mayoría de los casos entorpece el aprendizaje universitario, ya que los estudiantes que emplean de manera excesiva el uso del smartphone, incrementa la procrastinación y disminuye el nivel de rendimiento académico. Sin embargo, también identificaron estudiantes que manejan de forma óptima el internet, se 
reducen los tiempos de procrastinación, y a su vez, se incrementa su aprendizaje si existe una selección adecuada de los recursos tecnológicos que emplea para la construcción de conocimientos tanto de manera individual como de forma colaborativa.

El teléfono celular se puede emplear de manera positiva en el aula, ya que como Coll (2013) afirmó, la tecnología viene a ser parte de la nueva ecología del aprendizaje, por lo tanto, la existencia de la nomofobia entre los estudiantes es inherente. Sin embargo, los estudiantes pueden no estar preparados para utilizarlo de forma racional y caer en adicción que les perjudique su rendimiento académico.

\section{CONCLUSIÓN O CONSIDERACIONES FINALES}

Las implicaciones que ha traído el uso excesivo del celular, ha repercutido en el desempeño académico de los estudiantes, así como en su bienestar socio-emocional porque presentan síntomas como malestar, dolor de cabeza y ansiedad al encontrarse lejos de su dispositivo móvil. Se reconoce que el uso excesivo los ha alejado de su realidad, del contexto y de la gente cercana porque prefieren establecer comunicación de manera presencial que de forma virtual. Así como la nomofobia, existen otros fenómenos emergentes asociados con el celular, que valdría la pena abordar en futuros estudios porque aportarían una explicación a este nuevo ecosistema digital y su impacto directo en el proceso formativo de los estudiantes, en su desarrollo cognitivo, consecuente con el menoscabo de habilidades sociales presenciales. Las limitaciones de la investigación están relacionadas con otras variables, faltó entrevistar a profesores y padres de familia, es posible que sus reflexiones abonarían a profundizar en las dimensiones Físico-sociales y Emocionales.

\section{LISTA DE REFERENCIAS}

Asociación de Internet MX (2017). 13 Estudio sobre los hábitos de los usuarios en internet en México 2017. [En línea]. Disponible en: https://www.asociaciondeinternet.mx/es/estudios. Fecha de consulta: 19 de octubre de 2020.

Asociación de Internet MX (2018). $14^{\circ}$ Estudio sobre los Hábitos de los usuarios de Internet en México. [En línea]. Disponible en: https://www.asociaciondeinternet.mx/es/estudios. Fecha de consulta: 10 de noviembre de 2020. 
Britos, M. y Brítez, R. (2015). Uso y acceso a internet, teléfono celular y redes sociales en estudiantes paraguayos de la carrera de Psicología UCSA, 2015. Revista Científica de la UCSA. 2(2): 63-74.

Chou, H. and Edge, N. (2012). They Are Happier and having Better Lives than I Am the impact of using Facebook on Perceptions of others lives. Cyberpsychology, Behavior and Social Networking. 15(2): 117-121.

Coll, C. (2013). La educación formal en la nueva ecología del aprendizaje: Tendencias, retos y agenda de investigación. En J. L. Rodríguez-Illera (Ed.), Aprendizaje y educación en la sociedad digital (pp. 156-170). Barcelona: Universitat de Barcelona.

Dixit, S., Shukla, H., Bhagwat, A. K., Bindal, A., Goya, A., Zaidi, A. K., and Shrivastava, A. (2010). A study to evaluate mobile phone dependence among students of a medical college and associated hospital of central India. Indian Journal of Community Medicine. 35(2): 339-341.

Echeburúa, E. y Corral, P. (2010). Adicción a las nuevas tecnologías y a las redes sociales en jóvenes: un nuevo reto. Adicciones. 22(2): 91-96.

Flores, C., Gamero, K., Arias, W., Melgar, C., Sota, A. y Ceballos, K. (2015). Adicción al celular en estudiantes de la Universidad Nacional de San Agustín y la Universidad Católica de San Pablo. Revista de Psicología. 5(2): 13-25.

Forgays, D. K., Human, I., and Schreiber, J. (2014). Texting everywhere for everything: Gender and age differents in cell phone etiquette and use. Computers in Human Behavior. 31: 341-321.

García, V. and Fabila, M. A. (2014). Nomofilia vs. Nomofobia, irrupción del teléfono móvil en las dimensiones de la vida de los jóvenes. Un tema pendiente para los estudios de comunicación. Razón y Palabra. 18(86): 1-19.

González-Cabrera, J., León-Mejía, A., Pérez-Sáncho, C. y Calvete, E. (2017). Adaptación al español del cuestionario Nomophobia Questionnaire (NMP-Q), en una muestra de adolescentes. Actas Esp Psiquatr. 45(4): 137-144.

Jasso-Medrano, J. L., López-Rosales, F. y Díaz-Loving, R. (2017). Conducta adictiva a las redes sociales y su relación con el uso problemático del móvil. Acta de Investigación Psicológica. 7(3): 2832-2838. 
Junquera, C. (2016). Desconectados del entorno y conectados a la red: tan cerca pero tan lejos. KuberÉtica. [En línea]. Disponible en: https://www.kubernetica.com/2015/01/13/desconectados-del-entorno-yconectados-a-la-red-tan-cerca-pero-tan-lejos/. Fecha de consulta: 19 de junio de 2021.

King, A. L. S., Valença, A. M., and Nardi, A. E. (2010). Nomophobia: The mobile phone in panic disorder with agoraphobia: Reducing phobias or worsening of dependence? Cognitive and Behavioral Neurology. 23(1). 52-54.

King, A. L. S., Valença, A. M., Silva, A. C., Sancassiani, F., Machado, S., and Nardi, A. E. (2014). Nomophobia: Impact of cell phone use interfering with symptoms and emotions of individuals with panic disorder compared with a control group. Clinical Practice and Epidemiology in Mental Health: CP \& EMH. 10: 28-35.

Matoza-Baez, C. y Carballo-Ramírez, M. S. (2016). Nivel de nomofobia en estudiantes de medicina de Paraguay, Año 2015. CIMEL: Ciencia e Investigación Médica Estudiantil Latinoamericana. 21(1) 28-30.

Morales, J., Berrospi, H., Campos, B., Castro, M. y Sanchez, A. (2016). Nivel de nomofobia y rendimiento académico de los estudiantes de la universidad San Pedro sede Chimbote. Universidad San Pedro de Perú. [En línea]. Disponible en: http://repositorio.usanpedro.edu.pe/bitstream/handle/USANPEDRO/309/PI1640 431.PDF?sequence=1\&isAllowed=y. Fecha de consulta: 12 de abril de 2021.

Osio, A. M., Yucra, G., Arroyo, K., Berduguez, P., Ramírez, V., Reinaga, H., ... y López, M. C. (2014). ¿Es la nomofobia, un problema del Siglo XXI? Archivos Bolivianos de Medicina. 22(90): 56-63.

Pascual, M. F. (2015). Implementación del programa "conectar igualdad" en dos escuelas secundarias de Rosario. Invenio. 18(35): 105-122.

Quesada, V. J. y Carballo, I. C. (2017). Nomofobia, ¿qué es? Cuadernos de Atención Primaria. 23(1): 37-39.

Ramírez, K. M. y Vargas, I. A. (2018). Nomofobia y rasgos de personalidad en estudiantes de una universidad privada de Lima Metropolitana. Tesis de Licenciatura. [En línea]. Disponible en: http://repositorio.urp.edu.pe/bitstream/handle/URP/1922/1NOMOFOBIA\%20Y \%20RASGOS\%20DE\%20PERSONALIDAD\%20EN\%20ESTUDIANTES\%20 
DE\%20UNA\%20UNIVERSIDAD\%20PRIVADA\%20DE\%20LIMA\%20MET $\underline{\text { ROPOLITAN.pdf?sequence }=1 \& \text { isAllowed }=y} . \quad$ Fecha de consulta: 2 de noviembre de 2020.

Ramos-Galarza, C., Jadán-Guerrero, J., Paredes-Núñez, M., Bolaños-Pasquel, M. y Gómez-García, A. (2017). Procrastinación, adicción al internet y rendimiento académico de estudiantes universitarios ecuatorianos. Estudios Pedagógicos. 43(3): 275-289.

Ramos-Soler, I., López-Sánchez, C. y Quiles-Soler, M. C. (2017). Adaptación y validación de la escala de nomofobia de Yildirim y Correia en estudiantes españoles de la educación secundaria obligatoria. Salud y Drogas. 17(2): 201213.

Ruiz-Palermo, J., Sánchez-Rodríguez, J. y Trujillo-Rorres, J. M. (2015). Utilización de internet y dependencia a teléfonos móviles en adolescentes. Revista Latinoamericana de Ciencias Sociales, Niñez y Juventud. 14(2): 1357-1369.

Sánchez-Carbonell, X., Beranuy, M., Castellana, M., Chamarro, A. y Oberst, U. (2008). La adicción a Internet y al móvil: ¿moda o trastorno? Adicciones. 20(2): 149160.

SPSS, Statistical Package for the Social Sciences (2018). Statistics for Windows, (Versión 21.0.) [Software de cómputo estadístico para ciencias sociales]. Armonk, NY. Estados Unidos: IBM Corp. Released 2012.

Torres-Salazar, Q., Ramírez-Gurrola, A., Castañón-Alvarado, M., Aroña-Campos, A., Betancourt-Araujo, A., Carrillo-Cisneros, M. F., ..., y de-la-Cruz, B. C. (2018). Asociación entre nomofobia e indicadores de inteligencia en estudiantes de educación superior. Revista Internacional de Investigación de Adicciones. 4(2): $19-24$.

Yildirim, C. (2014). Exploring the dimensions of nomophobia: Developing and validating a questionnaire using mixed methods Research. Published Theses. IOWA University. [En línea]. Disponible en: https://lib.dr.iastate.edu/cgi/viewcontent.cgi?article=5012\&context=etd. $\quad$ Fecha de consulta: 15 de enero de 2020. 
Yildirim, C. and Correia, A. P. (2015). Exploring the dimensions of nomophobia: Development and validation of a self-reported questionnaire. Computers in Human Behavior. 49: 130-137. 